\title{
Ärztinnen in die Führung!
}

\section{Sibyl Schädeli}

MA, College M

\author{
Der Frauenanteil in der Schweizer Ärzteschaft steigt weiter. Seit zehn Jahren \\ schliessen mehr Frauen als Männer ein Studium der Humanmedizin ab. Nur mehr \\ bei den über Vierzigjährigen stellen die männlichen Ärzte noch die Mehrheit, bei \\ den jüngeren Jahrgängen sind es durchgängig Frauen. Diese neue Geschlechterver- \\ teilung setzt sich allerdings bei den ärztlichen Führungspositionen bisher nur \\ langsam durch: Lediglich 22\% der leitenden Arzt- und 11\% der Chefarztpositionen \\ waren 2014 von Frauen besetzt. Man spricht hier vom Phänomen der «leaky pipe- \\ line». Während also die Medizin weiter «feminisiert» wird, bleiben die Chefpositio- \\ nen in männlicher Hand?
}

\section{Feminisierung}

Unter dem Begriff «Feminisierung der Medizin» verstecken sich unterschiedliche Aussagen und Besorgnisse: Der Frauenanteil in der Medizin nimmt zu, damit aber auch der Anteil an Teilzeitbeschäftigten, Aussteigerinnen und Ärztinnen, die sich keine höhere Führungsfunktion vorstellen oder leisten können. Wichtige Ressourcen für Patienten und Patientinnen, Spitäler, aber auch das Gesundheitssystem insgesamt, bleiben damit ungenutzt. Aber auch individuelle Möglichkeiten, Qualitäten und Talente werden nicht ausge-

Résumé

La proportion de femmes dans le corps médical suisse continue d'augmenter. Depuis dix ans, les femmes sont plus nombreuses que les hommes à terminer des études de médecine humaine. Les médecins hommes ne sont plus majoritaires que chez les plus de 40 ans, les femmes dominent dans toutes les promotions plus jeunes. Cette nouvelle répartition des sexes ne s'impose toutefois que lentement au sommet de la hiérarchie: seuls $22 \%$ des postes de médecin-adjoint et $11 \%$ des postes de médecin-chef étaient occupés par des femmes en 2014. On parle ici du phénomène de "leaky pipeline», ou tuyau percé. Alors que la médecine continue de se féminiser, les positions de chef restent donc aux mains des hommes? schöpft. Während auf der einen Seite in dunklen Tönen ausgemalt wird, dass die sogenannte Feminisierung zu einer Statusminderung des ärztlichen Berufes führen wird, zeigen neue Studien, dass Patientinnen und $\mathrm{Pa}$ tienten von Ärztinnen profitieren, weil sich diese im Unterschied zu ihren männlichen Kollegen enger an Guidelines halten und besser kommunizieren würden. Wie eine neue Analyse (JAMA Internal Medicine 2016) zeigt, hatten Patientinnen und Patienten, die von einer Frau behandelt wurden, ein 4 Prozent tieferes (relatives) Risiko, innerhalb von 30 Tagen nach Spitaleintritt zu sterben, und ein 5 Prozent kleineres Risiko, in dieser Zeit erneut hospitalisiert zu werden. Anders ausgedrückt, hätten 233 bzw. 182 Patienten von einer Frau statt einem Mann behandelt werden müssen, um einem vorzeitigen Todesfall bzw. einer Rehospitalisierung zu entgehen. Dieser Zusammenhang war bei acht häufigen Krankheiten und verschiedenen Schweregraden der Erkrankung nachweisbar.

Es wäre zu kurz gegriffen, aus diesen widersprüchlichen Aussagen abzuleiten, dass der wachsende Frauenanteil in der Medizin gut für die Patientinnen und Patienten und schlecht für die männlichen Kollegen wäre. Das Problem ist vielschichtiger. So sind beispielsweise heute nicht nur Frauen an einer guten Balance von privatem und beruflichem Leben interessiert, sondern vermehrt auch Männer. Für beide Geschlechter, für Frauen allerdings noch weniger, scheint das Ziel Chefarzt oder Chefärztin keine grosse Attraktivität mehr zu haben (H. van den Bussche 2014). Für die vergleichsweise geringe Zahl an Ärztinnen in den oberen Führungspositionen gibt es verschiedene Ursachen. 
Neben fehlenden strukturellen Anpassungen im Bereich von Arbeitsmodellen, Kinderbetreuungsangeboten und weiteren Rahmenbedingungen spielen auch kulturelle und sozialisationsbedingte Faktoren eine wesentliche Rolle: Welches Klima, welche Umgangsformen herrschen in den Chefetagen? Wie schaffen es Frauen, frühzeitig ihre Fähigkeiten und Führungsqualitäten wahrzunehmen und nach aussen auch entsprechend zu verkaufen?

\section{Stolpersteine}

«Frau Dr. M. ist 34 Jahre alt, kinderlos, Oberärztin in einem Universitätsspital in der Schweiz. Sie hat erfolgreich studiert und ihr Staatsexamen mit Höchstnoten abgeschlossen. Sie arbeitet viel, leistet mehr Überstunden als ihre Kolleginnen, die bereits Kinder haben, und auch mehr als die meisten ihrer männlichen Kollegen.

\section{Die Mädchen-Erfolgsstrategien aus}

Schul- und Studienzeit funktionieren nicht in hierarchischen Institutionen.

Über ihre Leistungen hingegen spricht sie nicht besonders gern. Sie ist der Überzeugung, dass Fachkompetenz und Einsatz zum Erfolg führen. Zu Sitzungen erscheint sie in letzter Sekunde. Sie meldet sich nur zu Wort, wenn sie etwas fachlich Relevantes zu sagen hat. Eifrig schreibt sie Notizen und nimmt auch bereitwillig Aufträge entgegen, für die andere keine Zeit haben. Nach den Sitzungen verschwindet sie gleich wieder. Schliesslich hat sie noch viel abzuarbeiten. Apéros, Weihnachtsanlass und Skitag meidet sie nach Möglichkeit. Man wird schliesslich nicht fürs Herumstehen befördert. Ihren Kollegen und Kolleginnen gegenüber ist sie hilfsbereit und übernimmt schon mal eine Schicht oder ein paar Patienten. Für Projekte oder sonstige Gremien fehlt ihr die Zeit. Eines Morgens jedoch bricht ihre Welt zusammen: Kollege Dr. T., immer etwas lässig und eher faul, wird überraschend zum leitenden Arzt befördert. Sie kennt ihn bereits aus dem Studium. Ein ganz Netter, aber fachlich eindeutig Durchschnitt. Seine Frau erwartet gerade ihr zweites Kind, eine Beförderung kommt also zum richtigen Zeitpunkt. Verletzt und wütend verlangt sie ein Gespräch mit dem Klinikdirektor. Sie möchte wissen, weshalb sie nicht für die Stelle in Betracht gezogen wurde. Der Klinikdirektor ist erstaunt. Er hätte nie gedacht, dass Frau Dr. M. im Moment an einem Karriereschritt interessiert sein könnte. Sie beteiligt sich kaum an übergreifenden Projekten, ist schlecht vernetzt und scheint soziale Aktivitäten zu meiden. Sie wirkt stets an der Grenze der Überarbeitung. Luft nach oben scheint es

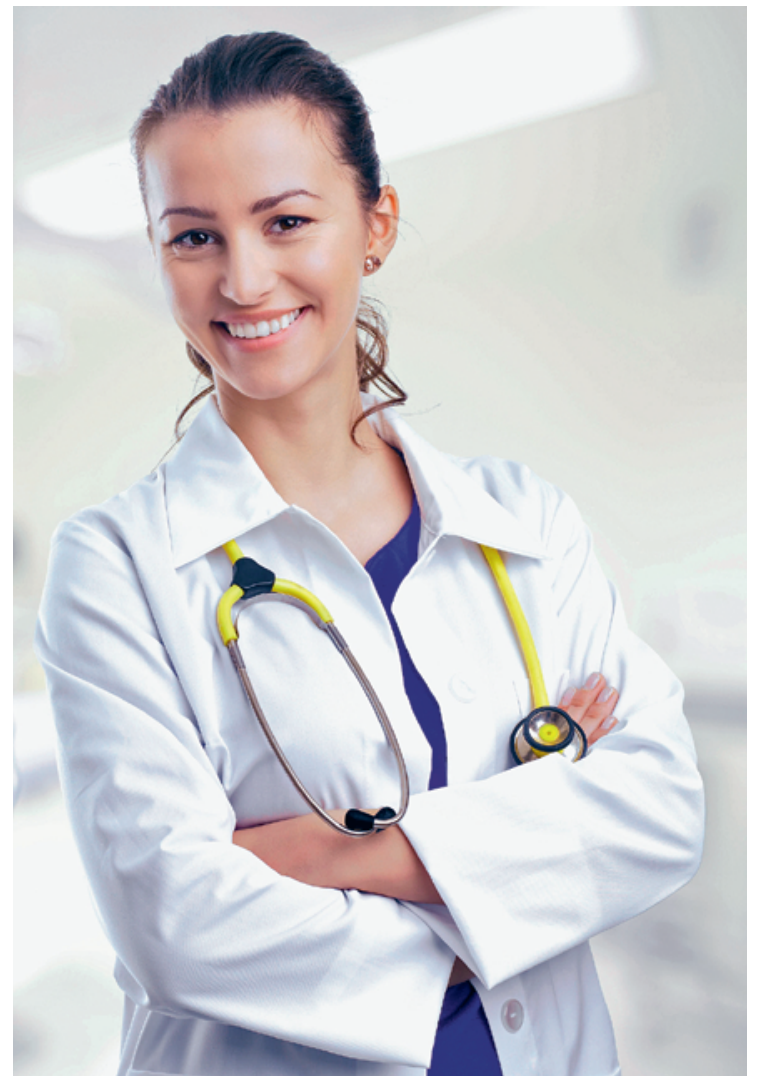

Obwohl seit zehn Jahren mehr Frauen als Männer das Studium der Humanmedizin abschliessen, sind nur 22\% der leitenden Arzt- und $11 \%$ der Chefarztpositionen (Stand 2014) von Frauen besetzt. Woran liegt das?

nicht zu geben. Vielleicht möchte sie ja noch Kinder? Kollege Dr. T. hingegen war letztes Jahr an einer Führungsweiterbildung, kennt alle wichtigen Entscheidungsträger im Spital, und sein Name steht als Co-Autor bereits auf mehreren Publikationen. Er spricht oft über seine Erfolge und hat seinen Führungsanspruch schon mehrfach angemeldet. Gemeinsam mit anderen Ärzten spielt er einmal in der Woche Tennis. Hier werden gelegentlich auch Absprachen und Entscheidungen getroffen, für die die offizielle Sitzung einfach nicht der passende Ort wäre. Der Klinikdirektor hat Dr. T. dabei als guten Unterstützer, aber auch als originellen Querdenker erlebt.»

Es ist aber nicht damit getan, dass sich Frauen nur den Gepflogenheiten der männlichen Karriereleiter anpassen.

Geschichten wie die von Frau Dr. M. gibt es zuhauf. Die Mädchen-Erfolgsstrategien aus Schul- und Studienzeit funktionieren nicht in hierarchischen Institutionen. Die stille Fleissige wird nicht entdeckt, die Prinzessin nicht wachgeküsst. Wer Bühnen meidet, strategisches Networking und Selbstmarketing unterschätzt und 
informelle Treffen auslässt, geht leer aus bei Beförderungen. Befördert wird man nicht dafür, dass man den Job macht, sondern dafür sorgt, dass er gemacht wird: «Get the job done!». Durchsetzungskraft, Auftrittsstärke, Allianzen und ein nötiges Mass an Lockerheit machen Karriere. Pech gehabt, Dornröschen!

Leider geht dadurch dem Gesundheitswesen ein beträchtliches Potential an hochkompetenten Führungsfrauen verloren. Dies, weil Kandidatinnen oftmals die Spielregeln nicht kennen, aber auch weil die Chefs die Sprache der begabten Frauen nicht verstehen. Hier ist grosser Bedarf an Öffnung und Verständnis für die kulturellen Unterschiede beiderseits gefragt. Es ist aber nicht damit getan, dass sich Frauen nur den Gepflogenheiten der männlichen Karriereleiter anpassen. Ihr Weg ist noch um eine Dimension komplexer. Dazu ein zweites Beispiel:

«Frau Dr. B. ist in einem Kantonsspital Assistenzärztin im letzten Jahr. Sie hat bereits Aussicht auf eine interessante Oberarztstelle in derselben Klinik. Hocherfreut erzählt sie davon ihren Kolleginnen und Kollegen. Von den Kollegen kommen nicht viele Reaktionen,

\section{Eine Führungskarriere zu machen bedeutet für Frauen eine schmale Gratwanderung zwischen zwei Minenfeldern.}

gelegentlich aber mal eine Gratulation oder ein Schulterklopfen. Die Kolleginnen hingegen reagieren säuerlich. Offenbar wieder eine, die meint, sie sei «etwas Besseres». Ihr Aufstieg hat wohl eher mit ihrem guten Aussehen als mit ihren Qualifikationen zu tun. Ständig treibt sie sich in der Nähe ihrer männlichen Kollegen herum, letztes Jahr war sie gar als Einzige mit einer Gruppe Kollegen beim Chefarzt eingeladen. Kinder scheinen sie nicht zu interessieren. Eine richtige Karrierefrau halt. In der Pause wechseln die Frauen nun gern das Gesprächsthema, wenn Frau Dr. B. dazukommt, und letzte Woche hat ihr niemand gesagt, dass ein Kinoabend geplant war.»

Frauen, die offen zu ihren Karriereabsichten stehen und einen lockeren Umgang mit ihren männlichen Kollegen pflegen, sind in Frauengruppen nicht gern gesehen. Studien belegen, dass eine Frau, die neu in eine Frauengruppe kommt, bereits nach durchschnittlich vier Minuten angegriffen wird. Nur wenn sie zeigen kann, dass sie sich einordnet und nicht «besser sein» will als die Kolleginnen, wird sie akzeptiert - bis zu dem Zeitpunkt, an dem sie einen Karriereschritt macht. Frauen gehen so weit, ihren besten KolleginnenFreundinnen Karriereerfolge zu verheimlichen. Sie leugnen gar, zu bestimmten Anlässen, Tagungen oder wichtigen Projekten eingeladen worden zu sein, aus Angst vor Repressionen. Und dieses Verhalten ist wiederum Gift in einem Umfeld, in dem Selbstmarketing von grösster Bedeutung ist.

\section{Eine Gratwanderung}

Eine Führungskarriere zu machen bedeutet für Frauen eine schmale Gratwanderung zwischen zwei Minenfeldern. Was immer ihnen auf der einen Seite gelingt, kann ihnen auf der anderen Seite zum Verhängnis werden. Verhalten sie sich «weiblich», drohen sie nicht befördert zu werden, verhalten sie sich «männlich», laufen sie Gefahr, von ihren Geschlechtsgenossinnen ausgegrenzt zu werden. Zum einen müssen sie also lernen, mit den (tendenziell männlich geprägten) «Spielen» der Organisationen einen adäquaten Umgang zu finden, und zum anderen müssen sie souverän auf die Gruppendynamik ihrer Kolleginnen reagieren. Es ist aber auch Aufgabe des Umfelds, hier wichtige Beiträge zu leisten. Wenn man im Gesundheitswesen erkennt, wie wichtig die Mehrheit der jungen Fachkräfte, die Ärztinnen, für die Zukunft der Medizin ist, dann sollte den erschwerten Bedingungen der Frauen bewusst Rechnung getragen werden. Und es braucht auch Mentoren und Mentorinnen, die sich mit Herzblut für Ärztinnen auf dem Karriereweg einsetzen!

\footnotetext{
Literatur

- van den Bussche H, Gedrose B. Bedingungen für Karrieren von jungen Ärztinnen und Ärzten. In: Ministerium für Innovation, Wissenschaft und Forschung des Landes Nordrhein-Westfalen (Hg): Gender-Kongress 2014. Tagungsdokumentation Essen 7. 3. 2014. Düsseldorf 2014, S. 38-42.

- Yusuke Tsugawa, Anupam B. Jena, Jose F. Figueroa et al (2016): Comparison of Hospital Mortality and Readmission Rates for Medicare Patients Treated by Male vs Female Physicians. In: Jama Internal Medicine, December 2016
}

Bildnachweis

(c) Sepy67| Dreamstime.com 